\title{
Salaires des assistantes médicales pour 2008 et recommandations de salaires
}

Etant donné que, depuis 1991, les sociétés cantonales de médecine élaborent leurs propres recommandations de salaires pour les assistantes médicales, nous renonçons désormais à publier des recommandations salariales pour l'ensemble de la Suisse.

Les conditions-cadres ci-après conservent toutefois leur valeur pour fixer individuellement le salaire d'une assistante médicale.

\section{Formation}

- Diplôme de la Fédération des médecins suisses (estampille) resp. certificat de capacité de la FMH avec certificat de radioprotection et autorisation de faire des radiographies;

- Certificat fédéral de capacité d'assistante médicale.

\section{Conditions de travail: bases de calcul}

- Semaine de 42 heures en moyenne annuelle;

- 4 semaines de vacances (assistantes médicales de moins de 20 ans et de plus de 50 ans: 5 semaines).

Toute dérogation importante à ces conditions sera répercutée sur le salaire.

\section{Normes régionales}

Les salaires doivent être adaptés aux conditions régionales.

\section{4. $13^{\mathrm{e}}$ salaire}

Un $13^{\text {e }}$ salaire doit être versé à l'assistante médicale à la fin de l'année. Si les rapports de service n'ont pas duré pendant toute l'année civile, le $13^{\mathrm{e}}$ salaire sera versé au prorata des mois d'engagement.

\section{Prime d'ancienneté et augmentation du salaire réel}

Les salaires étant bloqués maintenant depuis cinq ans, nous recommandons une augmentation du salaire réel de 1\%. Le montant de la prime d'ancienneté devrait figurer, également en 2008, parmi les points discutés au cours de l'entretien annuel sur les qualifications de l'employée.

\section{Compensation du renchérissement}

Le renchérissement devrait être compensé (ISPC septembre: 0,7\%; octobre: 1,3\%).

Lorsque la compensation du renchérissement est convenue par contrat, elle doit être accordée en tous les cas.

\section{Travail à temps partiel rétribué au mois}

Pour le travail à temps partiel, le salaire brut recommandé s'élève au $1 / 42$ du salaire brut entier multiplié par le nombre des heures de travail hebdomadaire convenu (base de calcul: 42 heures hebdomadaires).

\section{Salaire horaire}

Un salaire horaire est recommandé en cas $\mathrm{d}$ 'horaire de travail très réduit et en même temps irrégulier. Le taux horaire conseillé est de 6\%o du salaire mensuel (le $13^{\mathrm{e}}$ salaire y étant inclus au prorata). Il sera complété par une contribution aux vacances de 8,33\% correspondant aux quatre semaines de vacances habituelles (pour 5 semaines le taux sera de $10,64 \%$ et pour 6 semaines 13,04\%, etc.). Ce mode de calcul est également valable pour le paiement des heures supplémentaires.

\section{Heures supplémentaires}

La réglementation des heures supplémentaires a toujours fait l'objet de demandes de renseignements. Lors de la révision du contrat de travail au début de l'année 2001, ce point a été clarifié. Au chiffre 2 du contrat, il est désormais possible d'opter pour les solutions suivantes:

- La compensation des heures supplémentaires par du temps libre ou des vacances d'une même durée dont la date est décidée par l'employeur.

- L'indemnisation des heures supplémentaires par une majoration de salaire de $25 \%$. Le taux horaire est de $6 \%$, d'un plein salaire mensuel, auquel s'ajoute la contribution aux vacances (cf. chiffre 8 «Salaire horaire»).
Gaby Heimgartner

Secrétariat des délégués aux questions des assistantes médicales

Elfenstrasse 18

CH-3000 Berne 15 
Dans le cas d'une activité à temps partiel, les heures supplémentaires sont indemnisées selon le taux horaire normal, dans la mesure où elles ne dépassent pas la durée du travail réglementaire d'une assistante à temps plein au cabinet médical (cf. chiffre 2 du contrat).

\section{Les dispositions et recommandations suivantes s'appliquent à tous les salaires}

\section{Déductions sur le salaire brut}

- AVS, AI, APG, ACI: 6,05\% (AVS, AI, APG = $5,05 \%, \mathrm{ACI}=1 \%$;

- Assurance-accidents non professionnels (pour un engagement de plus de 8 heures par semaine): déduction selon le contrat d'assurance conclu;

- Prévoyance professionnelle (2e pilier LPP): part de l'employée à la contribution (habituellement 50\%) calculée en fonction de l'âge, selon le certificat d'assurance.

\section{Contrats de travail}

\section{et autres conditions d'engagement}

Nous préconisons d'établir le contrat par écrit, sur la formule de la FMH créée conjointement avec les associations des assistantes médicales, accompagnée des recommandations cantonales. Ces documents peuvent être obtenus aux adresses suivantes:

- Fédération des médecins suisses FMH, Elfenstrasse 18, 3000 Berne 15, tél. 03135911 11, e-mail: fmhmprax@hin.ch,www.fmh.ch;
- Association romande des assistantes médicales (ARAM), case postale 2034, 1002 Lausanne, tél. 02194345 64;

- Association suisse des assistantes médicales (SVA), case postale 6432, 3001 Berne, tél. 03138054 54, e-mail: sekretariat@sva.ch, www.sva.ch;

- Berufsverband Medizinischer Praxisassistentinnen (BMPA), Sonnenbergstrasse 7, 6005 Lucerne, tél. 04131022 23, e-mail: bsmpa@bluewin.ch, www.bsmpa.ch;

- Fédération suisse des associations d'assistantes médicales (FSAAM), chemin du Lussy 18, 1806 St-Légier, tél. 02194345 64, e-mail: fsaam-ch@bluewin.ch, www.fsaam. ch;

- Association genevoise des assistantes médicales (AGAM), rue des Pavillons 11, 1205 Genève, tél. 0223211069.

L'Association d'assistantes médicales du Tessin a son propre modèle de contrat de travail; son adresse est la suivante:

- Associazione Ticinese Assistenti di studio Medico (ATAM), Via Ronchetto, 6814 Cadempino, tél. 0919947835.

Les salaires des apprenties se basent sur les recommandations cantonales. 